\title{
Deep learning networks find unique mammographic differences in previous negative mammograms between interval and screen-detected cancers: a case-case study
}

Benjamin Hinton ${ }^{1,2^{*}}$ (D), Lin Ma ${ }^{3}$, Amir Pasha Mahmoudzadeh ${ }^{4}$, Serghei Malkov ${ }^{5}$, Bo Fan ${ }^{1}$, Heather Greenwood ${ }^{2}$, Bonnie Joe ${ }^{2}$ Vivian Lee ${ }^{6}$, Karla Kerlikowske ${ }^{7}$ and John Shepherd ${ }^{8}$

\begin{abstract}
Background: To determine if mammographic features from deep learning networks can be applied in breast cancer to identify groups at interval invasive cancer risk due to masking beyond using traditional breast density measures.

Methods: Full-field digital screening mammograms acquired in our clinics between 2006 and 2015 were reviewed. Transfer learning of a deep learning network with weights initialized from ImageNet was performed to classify mammograms that were followed by an invasive interval or screen-detected cancer within 12 months of the mammogram. Hyperparameter optimization was performed and the network was visualized through saliency maps. Prediction loss and accuracy were calculated using this deep learning network. Receiver operating characteristic (ROC) curves and area under the curve (AUC) values were generated with the outcome of interval cancer using the deep learning network and compared to predictions from conditional logistic regression with errors quantified through contingency tables.

Results: Pre-cancer mammograms of 182 interval and 173 screen-detected cancers were split into training/test cases at an 80/20 ratio. Using Breast Imaging-Reporting and Data System (BI-RADS) density alone, the ability to correctly classify interval cancers was moderate (AUC $=0.65$ ). The optimized deep learning model achieved an AUC of 0.82. Contingency table analysis showed the network was correctly classifying $75.2 \%$ of the mammograms and that incorrect classifications were slightly more common for the interval cancer mammograms. Saliency maps of each cancer case found that local information could highly drive classification of cases more than global image information.
\end{abstract}

Conclusions: Pre-cancerous mammograms contain imaging information beyond breast density that can be identified with deep learning networks to predict the probability of breast cancer detection.

Keywords: Breast Cancer, Masking, Mammography, Interval Cancer, Deep learning, Transfer learning, Neural network, Breast density

\footnotetext{
*Correspondence: bhinton@berkeley.edu; ben.hinton12@gmail.com

1 Department of Bioengineering, University of California-San Francisco

Berkeley Joint Program, Room A-C106-B, 1 Irving St, San Francisco, CA 94143, USA

${ }^{2}$ Department of Radiology and Biomedical Imaging, UC-San Francisco, San

Francisco, CA 94143, USA

Full list of author information is available at the end of the article
}

(c) The Author(s). 2019 Open Access This article is distributed under the terms of the Creative Commons Attribution 4.0 International License (http://creativecommons.org/licenses/by/4.0/), which permits unrestricted use, distribution, and reproduction in any medium, provided you give appropriate credit to the original author(s) and the source, provide a link to the Creative Commons license, and indicate if changes were made. The Creative Commons Public Domain Dedication waiver (http://creativecommons.org/publicdomain/zero/1.0/) applies to the data made available in this article, unless otherwise stated. 


\section{Background}

Breast cancer is a common disease with 1 in 8 women experiencing some form of malignant breast cancer in their lifetime [1]. In 2013, this translated to approximately 230,000 new cases of invasive breast cancer and 40,000 deaths in the US alone [1]. Detecting and treating breast cancer is extremely important for women's health and studies have shown that early detection of breast cancer yields higher survival rates [2].

Mammography is the current gold standard in screening for breast cancer in average-risk women. However, radiologically dense and complex tissue can reduce screening detection sensitivity leading to obscured breast lesions and cancers missed by screening mammography $[3,4]$. These cancers discovered within 12 months after normal screening mammograms are defined as interval cancers, and the reduction of mammographic sensitivity from breast density is commonly called masking. Roughly $13 \%$ of breast cancers diagnosed in the U.S. are interval cancers [5], and identifying women at high risk of interval cancers could prove useful to inform discussions on supplemental imaging.

Previous studies have shown that the Breast ImagingReporting and Data System (BI-RADS) breast density and other quantitative density measures are not only risk factors for breast cancer, but also for interval breast cancer risk due to the masking effect of radiologically dense breasts [4, 6]. While clinically measured BI-RADS breast density is a risk factor for interval cancer such that federal and state legislation has been passed to notify women of high BI-RADS breast density [7], the classification is subjective and does not account for the texture of dense tissue [8-10]. Because of this, the American College of Radiology has asked for development of direct measures of masking and interval risk [11].

Researchers have also leveraged imaging methods beyond conventional 2D digital mammography such as tomosynthesis [12, 13], MRI [14], and diffusion weighted (DW) MRI [15-17]. Within DW MR imaging, improvements have been made in lymph node assessment and risk of recurrence. Additionally, computer vision methods have been applied to mammography to identify masking risk. Previous studies have measured the ability of predefined kernels and model observers to quantify masking and interval cancer risk, indicating some promise in computer vision to identify interval cancer risk [10]. Advanced computer vision methods such as deep learning have shown promise in many computer vision tasks and have performed extremely well in the ImageNet competition compared to traditional pre-defined kernel methods [18, 19]. Transfer learning [20,21] of these networks has been effective in medical applications including breast cancer, where deep learning models were often able to equal or improve current classification or diagnostic schemes performed [22-27]. Another useful property of deep learning networks is their ability to highlight pixels containing unique information relevant to that image's classification called saliency maps, which can be used in biological applications to develop hypothesis on the underlying biology or features associated with the classification of interest $[26,28]$.

Deep learning has consistently been applied to and improved on current diagnostic methods in many medical fields [21, 25, 29], from lung pathology diagnosis [26] to identifying diabetic retinopathy [30, 31]. Deep learning methods have also been applied to a wide variety of areas of breast cancer research with promising results [32]. Deep learning has been applied to improve lesion detection in computer-aided detection [24], to identify and segment soft tissue lesions [33, 34], to identify and reduce potential false positives to reduce biopsies [35], to effectively categorize the amount of dense tissue in mammograms [36], and to improve lesion classification systems on breast tomosynthesis images [27]. Our study further aligns with these studies in their goals of using deep learning to improve breast cancer outcomes.

The purpose of this study was to implement a deep learning network to investigate if unique imaging characteristics exist beyond breast density, to classify precancerous mammograms that later result in either an interval or screen-detected invasive cancer within 12 months of the mammogram. We hypothesized that deep learning networks can more effectively quantify risk of interval cancer than BI-RADS breast density alone. If successful, these methods could be expanded to improve risk prediction models for interval cancer, develop automated methods or software that can aid radiologists in risk prediction, or to further understand radiomic quantities as they relate to underlying cancer biology.

\section{Methods \\ Participants}

Participants were selected from a screening population that had received full-field digital mammograms acquired from 2006 to 2015 from four radiology facilities, University of California - San Francisco, California Pacific Medical Center, Marin General Hospital, and Novato Community Hospital that participate in the San Francisco Mammography Registry. Ethics approval was obtained by the University of California - San Francisco Institutional Review Board for this retrospective analysis of mammograms. Invasive interval cancers from these institutions were included, defined as invasive cancers identified within 12 months of a negative screening examination. For interval cancers the negative mammogram prior to the mammogram leading to the eventual interval cancer diagnosis was chosen. An equal number of screen-detected cancers were matched by age and 
race if such matching data existed, based on all screendetected cancers diagnosed at the four centers. Screendetected cancers were defined as invasive cancers identified within 12 months of a positive screening examination. All mammograms were interpreted prospectively by radiologists during the course of routine clinical care. Cancers were identified by annual linkage to the state California Cancer Registry. Information was unavailable pertaining to the size of the lesions, specific cancer types, or whether the interval cancer was due to missed lesions or true interval cancers.

\section{Mammography}

The de-identified raw, "For Processing" representation of the standard four screening views (Mediolateral-oblique (MLO) and Cranio-Caudal (CC) images of both sides) were used for this study. All images were acquired on Hologic Selenia full-field digital mammography systems. These images were pre-processed in order to maximize the information provided to the network in the following ways. First, the skin edge of these images was identified and excess background of the images was cropped out using thresholding and in-house software [37]. The images were then normalized on a 0 to 255 scale. Various methods exist to input images from multiple views, from inputting images individually, to making separate networks for each view, to combining the images as a collage [38]. We implemented a collage and the four views were stacked as a $2 \times 2$ collage image for each case, with one view in each quadrant. This allowed all four views to be contained in a single image. This method has been performed in applications such as brain MRI slices to indicate Alzheimer's risk [38]. These images were then separated into a training and test set at an 80/20 split.

\section{Deep learning model}

An existing deep learning network architecture (ResNet50) was implemented with ImageNet transfer learning weights on all convolutional blocks [19]. A fully connected layer was then added with 256 weights, a dropout layer, and a final weight with sigmoid activation to classify between screendetected and interval cases. Figure 1 shows a diagram of the deep learning architecture and the fully-connected layer [19]. The weights of the fully-connected layer were randomly initialized and pre-trained on the training set images. During training, a binary cross entropy loss metric was optimized with a stochastic gradient descent optimizer. During model training, data augmentation was performed by introducing a random amount of shear, zoom, rotation, and horizontal and vertical reflection within specific ranges in order to increase the data variability and reduce overfitting. For each epoch, training loss, test loss, and accuracy were recorded and network weights were saved if it improved the test loss. The final network weights used were the weights with the best test loss throughout training.

Model hyperparameters for data augmentation, training parameters, and optimization parameters were selected through hyperparameter sweeps of a variety of hyperparameters. The hyperparameters were swept through a full realizable range of values for each parameter and loss and accuracy curves were examined to determine the range for each hyperparameter that resulted in a positive accuracy and loss trend as well as a small generalization gap between training and test data. Data

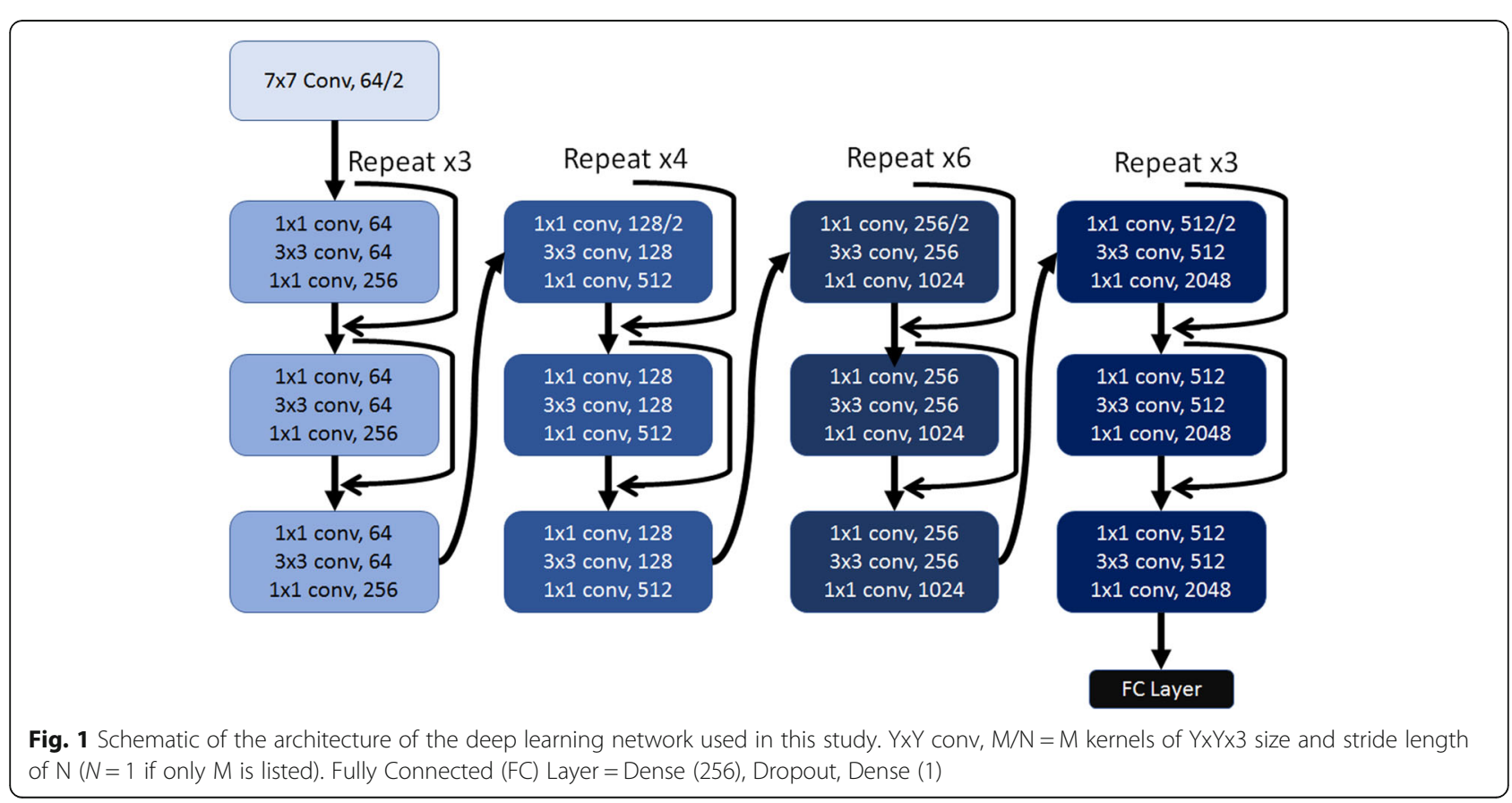


augmentation hyperparameters were rotation, zoom, shear, horizontal reflection, and vertical reflection and were applied to the training data. Training hyperparameters were learning rate, batch size, number of epochs, image input size, and number of convolutional layers to allow to re-train weights. Model optimizer hyperparameters were momentum, regularization, and decay. Loss and accuracy were computed in the training and test set. Saliency maps were produced along with a contingency table enumerating the number of correct and incorrect predictions with some sample images in order to understand what factors contributed to incorrect and correct predictions. Training was done on an NVIDIA K2200 GPU with 16 GB RAM. Image preprocessing was done in Matlab r2015a (Mathworks, Natick, MA), ResNet50 was implemented with Keras and Tensorflow [39] using Spyder 3.2.3 and Python 3.5.

\section{Model statistical testing}

After training was complete, conditional logistic regression was performed over the entire dataset in three cases: one with BI-RADS density as a classifier, one with the deep learning network predictions as a classifier, and one with both. In all cases interval vs. screen-detected breast cancer were the two outcomes. Receiver operating characteristic (ROC) curves were produced with area under the curve (AUC) values in all cases and compared. Statistical analysis and figure generation was performed via Spyder and $\mathrm{R}$ version 3.2.2.

\section{Results}

A total of 316,001 examinations on were performed in the screening population, leading to a total of 245 interval cancers of which 182 women were available for this study. Table 1 shows the demographic information of the women from each case-type. These were matched by age and race to 173 women with screen-detected breast cancers. There were no screen-detected cancers that matched by age and race for 9 of the interval cancers. These were included in the deep learning training to maximize the dataset, but were excluded in the conditional logistic regressions to ensure matching. The descriptive statistics showed a lower body mass index (BMI) and higher proportion of women with dense

Table 1 Descriptive statistics of the screen-detected and interval cancer groups. Percentage in each BI-RADS category are calculated excluding the missing/unknown groups

\begin{tabular}{|c|c|c|c|}
\hline & Screen-Detected Group & Interval Group & $P$-Value \\
\hline N & 173 & 182 & \\
\hline Age, years (Standard Deviation) & $57.8(10.9)$ & $56.8(11.8)$ & 0.28 \\
\hline BMI, kg/m² (Standard Deviation) & $24.9(4.7)$ & $23.5(4.3)$ & $<0.0001$ \\
\hline Time to Detection (Days) & $56.3(81.4)$ & $239.8(94.6)$ & $<0.0001$ \\
\hline Race: & & & 0.88 \\
\hline White & 127 & 129 & \\
\hline African American & 3 & 4 & \\
\hline Chinese & 25 & 27 & \\
\hline Filipina & 3 & 3 & \\
\hline Hispanic & 0 & 2 & \\
\hline Japanese & 5 & 8 & \\
\hline Mixed & 5 & 5 & \\
\hline Other Asian & 2 & 1 & \\
\hline Other Non-Asian & 3 & 3 & \\
\hline Menopausal status & 119 (69\%) & $123(68 \%)$ & 0.69 \\
\hline Family history of breast cancer & $47(23 \%)$ & $60(33 \%)$ & 0.25 \\
\hline Previous history of breast biopsy & $55(32 \%)$ & $68(37 \%)$ & 0.33 \\
\hline BI-RADS Frequency: & & & 0.008 \\
\hline A: Almost Entirely Fatty & $11(7.8 \%)$ & $3(1.8 \%)$ & \\
\hline B: Scattered Fibroglandularities & $50(35.5 \%)$ & $33(19.7 \%)$ & \\
\hline C: Heterogeneously Dense & $61(43.3 \%)$ & $78(46.7 \%)$ & \\
\hline D: Extremely Dense & 19 (13.5\%) & $53(31.7 \%)$ & \\
\hline Missing Data & 19 & 7 & \\
\hline Unknown & 13 & 8 & \\
\hline
\end{tabular}


breasts in the interval compared to the screen-detected breast cancer group. All other demographic and risk information was similar between groups.

Table 2 shows the end results of the hyperparameter sweep and optimal hyperparameters that were used in training our network. Of note we learned moderately aggressive image augmentation hyperparameters controlled overfitting while still allowing learning to take place. Additionally, a large batch size improved training by introducing the optimizer to more data and a learning rate in the range of $1 \mathrm{e}^{-3}-1 \mathrm{e}^{-5}$ produced good learning results. High dropout in the final fully-connected layers helped to control overfitting as well. Optimal parameters were selected based on their ability to reduce overfitting based on the training and test loss. Training time on this system under these parameters was roughly $3 \mathrm{~h}$ per 500 epochs.

Figure 2 shows the loss and accuracy of the model over time and compares the result of the training and test set. We can see that the generalization gap between train and test loss is small and that the curves in the test and train set are similar. This indicates that classification results were similar in both training and test sets. The best test loss occurred in epoch 482, with a test loss of 0.499 and test accuracy of $75.2 \%$.

Figure 3 compares the classification ROC analysis and AUC of the deep learning network versus using just BI-RADS density in a conditional logistic regression, and a final analysis combining both of these methods. The deep learning network outperforms
BI-RADS density alone in predicting interval versus screen-detected cancer.

Table 3 shows a contingency table quantifying the number and percent of correct and incorrect predictions in each category. The algorithm performed similarly for both cancer types - screen-detected and interval breast cancers were classified into their correct categories 77 and $74 \%$ of the time, respectively. Seventy-five percent of the total images (268/355) were correctly categorized.

Figure 4 shows the pseudo presentation mammograms (produced using methods described by Malkov, et al. [40], saliency maps, and then the superposition of the two for representative screen-detected and interval mammogram visits, both of which were correctly classified. The intensity of the saliency signal is shown from 0 to 255 color scale. A threshold was applied to highlight the regions above the 50\% activation level in the network to improve image clarity. The side and quadrant (if available) where the cancer was found in subsequent mammograms is also shown. We observed that localized regions could highly influence the classification, but that broad regions of the breast could influence decision making as well.

\section{Discussion}

We developed a deep learning algorithm that provided better discrimination than BI-RADS breast density for classifying interval cancer versus screen-detected cancer with a $75 \%$ classification accuracy compared with $63 \%$

Table 2 Chosen hyperparameters with brief description. Hyperparameter sweep went through a realizable range for each hyperparameter and individual values were chosen to optimize training ability or to minimize overfitting, depending on the parameter

\begin{tabular}{|c|c|c|c|}
\hline Hyperparameter (Range) & $\begin{array}{l}\text { Hyperparameter } \\
\text { Type }\end{array}$ & Interpretation & Chosen Value \\
\hline Rotation (0-90) & Data Augmentation & Range for a random rotation & 20 \\
\hline Zoom (0-1) & Data Augmentation & Range for a random zoom & 0.5 \\
\hline Shear $(0-1)$ & Data Augmentation & Range for a random shear & 0.3 \\
\hline Vertical/Horizontal Flip (Yes/No) & Data Augmentation & Random chance of flip in respective direction & Yes/Yes \\
\hline Momentum (0-1) & $\begin{array}{l}\text { Optimizer } \\
\text { Parameter }\end{array}$ & Accelerates or dampens oscillations in given direction. & 0.3 \\
\hline Regularization (0-1) & $\begin{array}{l}\text { Optimizer } \\
\text { Parameter }\end{array}$ & Penalty applied to large image weights & 0 \\
\hline Decay $(0-1)$ & $\begin{array}{l}\text { Optimizer } \\
\text { Parameter }\end{array}$ & Learning Rate decay over each update. & $1 e-5$ \\
\hline Dropout (0-1) & $\begin{array}{l}\text { Fully-connected } \\
\text { Layer }\end{array}$ & $\begin{array}{l}\text { Percent of weights dropped out between dense layers in the FC } \\
\text { layer. }\end{array}$ & 0.95 \\
\hline Learning Rate (0-1) & Training Parameter & Importance attributed to weight updates. & $1 e-3$ \\
\hline Epochs (Integer) & Training Parameter & Number of epochs performed & 1000 \\
\hline Batch Size ( $2^{n}$ any $\left.n\right)$ & Training Parameter & Number of samples per gradient update & 16 \\
\hline Image Size (Minimum 224) & Training Parameter & Input image size in pixels & 224 \\
\hline $\begin{array}{l}\text { nLayersRetrain (Fully Connected only - All } \\
\text { Layers) }\end{array}$ & Training Parameter & Number of layers allowed to have their weights altered. & $\begin{array}{l}\text { All Layers } \\
\text { (173) }\end{array}$ \\
\hline
\end{tabular}



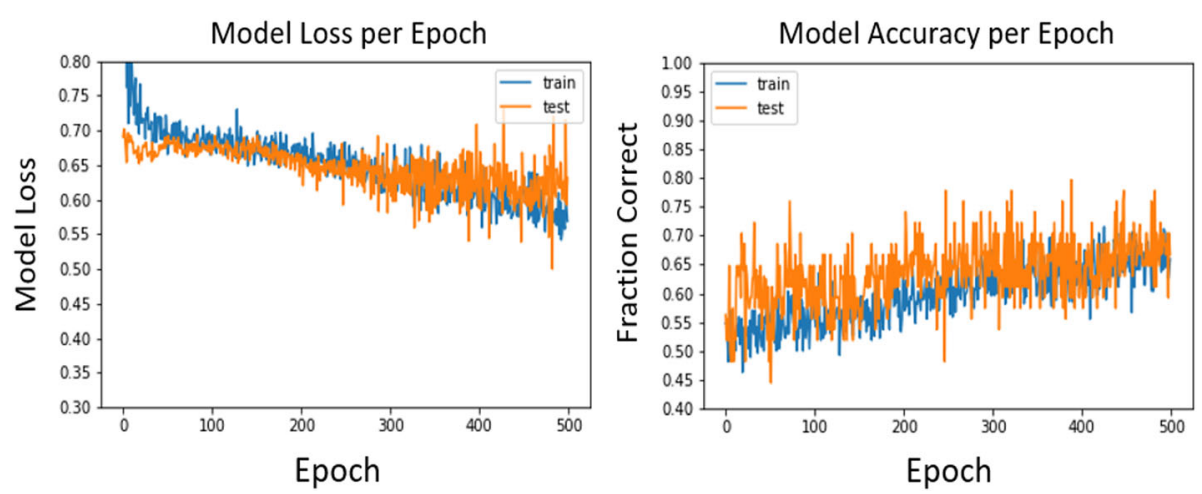

Fig. 2 Loss and accuracy curves per epoch of the test and train set of the deep learning network. Best test loss occurred on epoch 482 . At that epoch training loss and accuracy were 0.58 and $67.4 \%$, respectively, and test loss and accuracy were 0.499 and $75.2 \%$, respectively

for BI-RADS density. Deep learning networks have been applied in a variety of ways in breast cancer, but as of yet they have not been leveraged to identify risk of interval breast cancer. The results of our work indicate that a deep learning network is able to identify information in mammograms associated with interval breast cancer diagnosis that is not captured in the BI-RADS density classification alone.

Previous work by Kerlikowske et al. [4] showed that breast density was associated with increased prevalence of interval cancer in a screening population. Furthermore, Kerlikowske showed that using a combination of breast density and 5-year breast cancer risk to identify

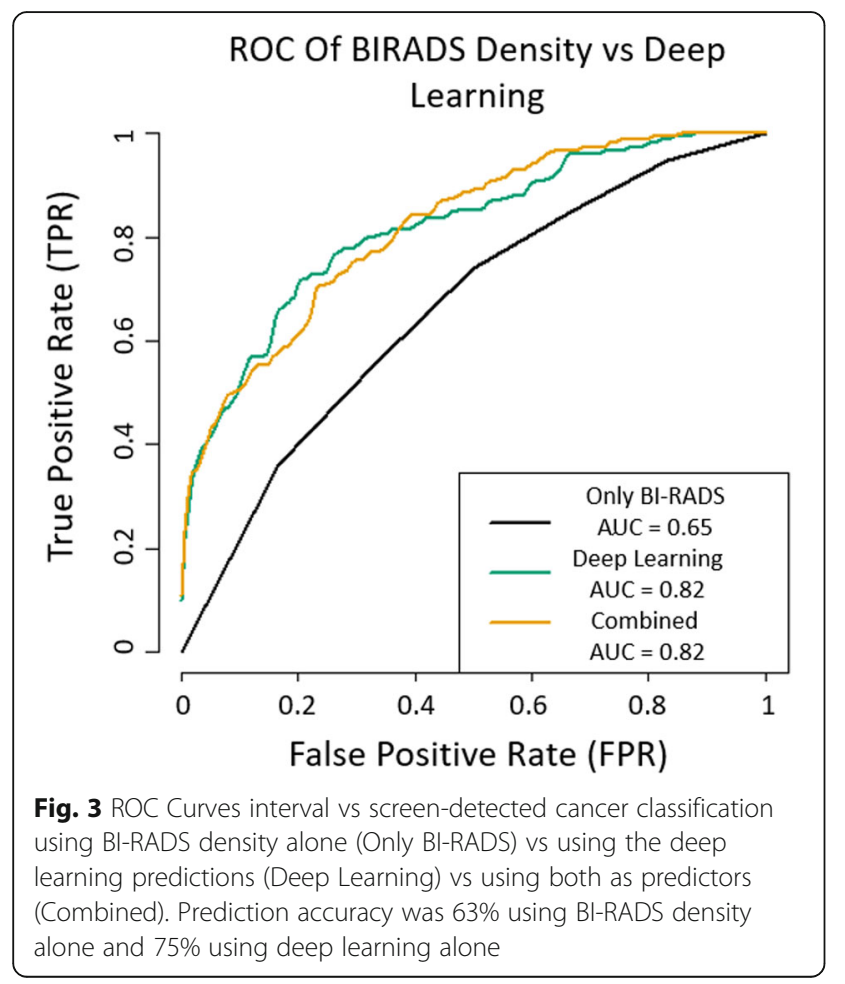

women for discussion about supplemental screening in the fewest women counseled per interval cancer occurrence. Recently, automated methods to quantify breast density have been shown to produce similar levels of interval risk as subjective BI-RADS density scores [6].

Other researchers have investigated radiomic features as a measure for interval risk. Strand et al. identified mammographic image features significant for interval breast cancer risk [9]. Holm et al. identified biological risk factors significant for interval risk after controlling for age and mammographic density [41]. Additionally, Mainprize et al. developed a direct measure of detectability that was significant for interval risk as well using model observers [10].

This study has several strengths. First, the dataset controls for age and race, helping to reduce possible confounding. Further, comparing our network to predictions based on BI-RADS density provides comparisons against current interval cancer risk factors [4, 7]. Additionally the transfer learning methods, the data preprocessing steps, data augmentation steps, and hyperparameter sweeps performed helped to maximize test accuracy.

Seventy-five percent of images analyzed were correctly classified, with slightly more actual interval images being misclassified compared to actual screen-detected images. This could be because the higher density of the interval images made them more difficult to classify. The saliency maps provided interesting information about the images and which regions influenced the decisions to classify the image as an interval or a screen-detected

Table 3 Contingency table of the number of correctly and incorrectly classified images from the deep learning network

\begin{tabular}{llll}
\hline Number (Percent) & Predicted Screened & Predicted Interval & Total \\
\hline Actual Screened & $134 / 173(77.4 \%)$ & $39 / 173(22.5 \%)$ & 173 \\
Actual Interval & $48 / 182(26.4 \%)$ & $134 / 182(73.6 \%)$ & 182 \\
Total & 182 & 173 & \\
\hline
\end{tabular}




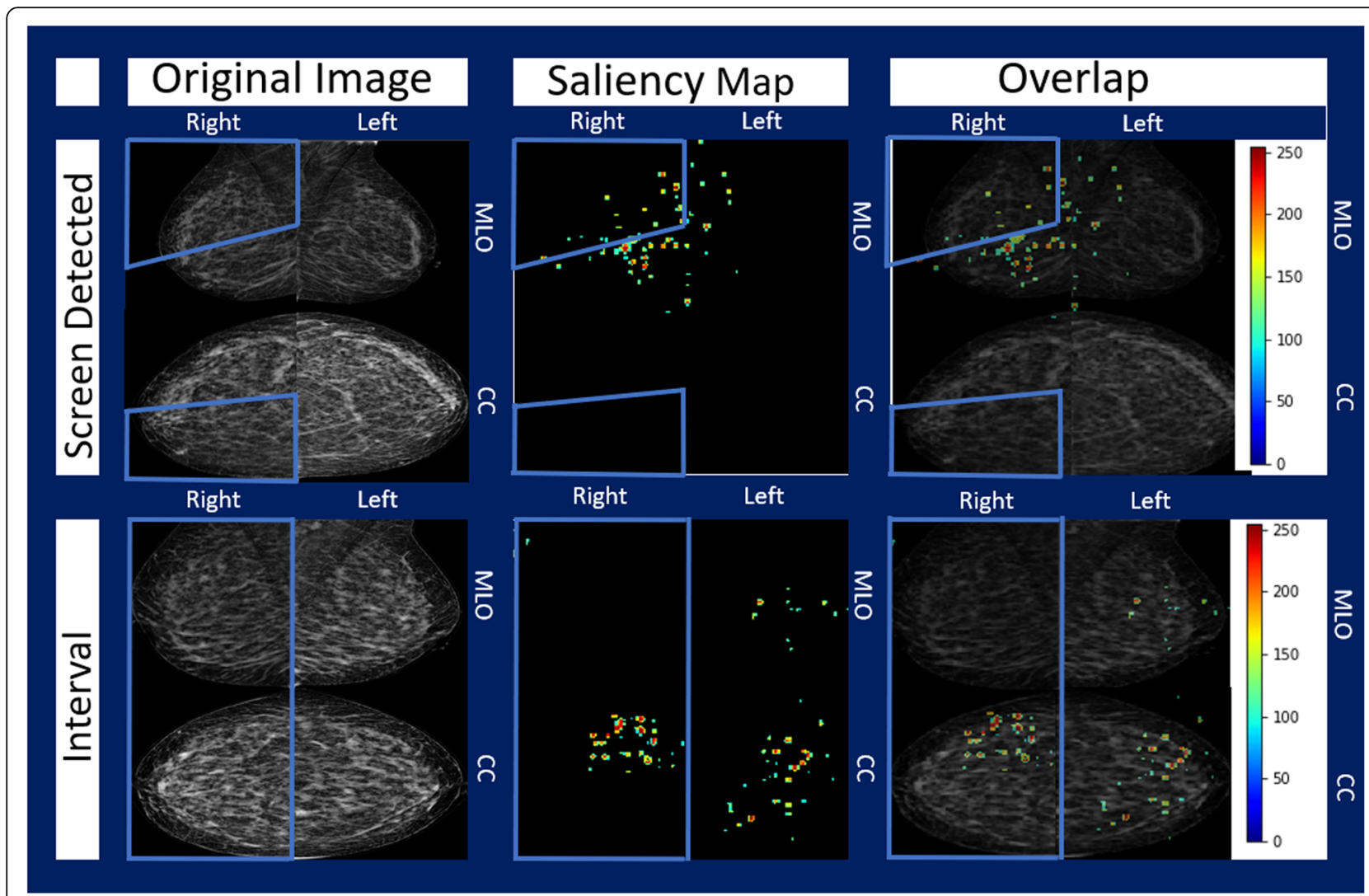

Fig. 4 Saliency maps of sample screen-detected and interval images (both correctly classified). For each row, the pseudo-presentation images are shown (left) along with the saliency map (middle) that highlights the pixels that had above a 50\% weight in classifying the image in its respective category (i.e. first row saliency map highlights weights that push towards decision of classifying as screen-detected decision). At right, the images are overlaid

image. While it appears regions of interest in the interval image were related to density, further work must be done to examine how these regions in the saliency maps relate to the underlying biological and radiomic features of the image. The goal of saliency maps like this is to help bridge the gap between the deep learning network predictions and the radiologist or interpreter, helping to identify regions at high risk of interval breast cancer or identify regions to study further regarding why and how they contribute to interval breast cancer risk.

There were several limitations to our study. First, the computational limitations of our system required a large amount of downsampling, which likely lost significant imaging details and textures. Future work should utilize more powerful systems capable of dealing with larger image sizes. Additionally, because of the limited number of cases available and our goal to use as much data as possible in training, we were not able to employ a validation dataset and we included some interval images without direct matches or images without BI-RADS density values. We attempted to mitigate the risks from having a smaller dataset through transfer learning, data preprocessing and augmentation, and careful selection of hyperparameters. Additionally, comparing results of the test and train sets to ensure they had similar results helped to identify hyperparameter sets that produced good training and test results.

Another limitation in our dataset was that it did not control for BMI or length of time between mammogram or diagnosis, leading to potential bias between the mammograms. Additionally, this initial study only compared against the subjective BI-RADS density, and did not compare against more quantitative measures of breast density. Previous work has shown that BI-RADS density and automated density measures were shown to be similar risk factors for interval cancer [7], but future work should include comparisons against automated density measures and other known masking features. Further, we did not have information regarding cancer types or lesion sizes, which would be useful information for future analyses. An additional limitation was that this study did not include a healthy control group that did not develop cancer. Our hypothesis was that there were fundamental differences in the mammograms of women that develop interval versus screen-detected cancers. We found a strong signal to confirm this hypothesis, guiding 
the path to future studies that will compare interval and screen detected cancers to women that do not develop breast cancer. Lastly, we used a broad definition of interval cancer and did not differentiate between interval cancers from missed lesions and true interval cancers, which may have led to the deep learning network achieving some of its performance by detecting certain visible features. While many interval cancers occur because the lesion is masked, some interval cancers occur due to radiologist fatigue or error and others from fast growing lesions that develop after the previous mammogram [42]. Identifying and separating these subgroups can be difficult. We did not separate these types of interval cancers, which introduced additional noise into the dataset and may have weakened our results compared to using a dataset of only truly masked interval cancers.

\section{Conclusions}

We conclude that pre-cancerous mammograms contain imaging information beyond breast density that can be used to predict the probability of breast cancer detection, and that deep learning models may be able to detect and identify that imaging information. This work could be expanded upon further to improve risk prediction models for interval cancer, develop automated methods or software that can aid radiologists in risk prediction, and understand if these deep learning predictions relate to underlying radiomic quantities or tissue biology.

\section{Abbreviations}

AUC: Area Under the Curve; BI-RADS: Breast Imaging - Reporting and Data System; BMI: Body Mass Index; CC: Craniocaudal; MLO: Mediolateral Oblique; ROC: Receiver Operating Characteristic

\section{Acknowledgements \\ The authors would like to thank the California Breast Cancer Research Program (CBCRP) for their generous grant that made this work possible under grant \#21IB-0130 and National Institutes of Health (NIH) Grant R01CA166945 and P01 CA154292 for support of collecting the raw digital images for the study. We would additionally like to thank the San Francisco Mammography Registry. We would also like to thank Vivian Lee and every advocate we have met in this research for their feedback and interest in this work. Further, the authors would like to thank the UCSF Breast Oncology Program Conference for providing settings in which to discuss this work. This material is based upon work supported by the National Science Foundation Graduate Research Fellowship Program under Grant No. 1144247. Any opinion, findings, and conclusions or recommendations expressed in this material are those of the authors and do not necessarily reflect the views of the National Science Foundation.}

\section{Authors' contributions}

BJH planned methods, preprocessed images, designed and turned deep learning networks, analyzed results, and wrote manuscript. LM organized mammogram images and demographic data. APM organized mammogram images and demographic data. SM wrote image preprocessing software. BF organized data and consulted on results. HG consulted on results, provided radiologist perspective, and edited manuscript. BJ consulted on results, provided radiologist perspective, and edited manuscript. VL edited manuscript and provided patient perspective. KK designed study, organized data, analyzed results, and edited manuscript. JS designed study, organized data, analyzed data \& results, edited manuscript. All authors read and approved the final manuscript.

\section{Authors' information}

Not Applicable.

\section{Funding}

The California Breast Cancer Research Program (CBCRP) for their generous grant that made this work possible under grant \#21IB-0130 and National Institutes of Health (NIH) Grant R01CA166945 and P01 CA154292 for support of collecting the raw digital images for the study. This material is based upon work supported by the National Science Foundation Graduate Research Fellowship Program under Grant No. 1144247. Any opinion, findings, and conclusions or recommendations expressed in this material are those of the authors and do not necessarily reflect the views of the National Science Foundation.

\section{Availability of data and materials}

The datasets used and/or analyzed during the current study are publicly available from the corresponding author on reasonable request.

\section{Ethics approval and consent to participate}

Ethics approval was obtained by the University of California - San Francisco Institutional Review Board for this retrospective analysis of mammograms. We have passive permission to use the risk factor, radiology, cancer and image data from this California Cancer Registry.

\section{Consent for publication}

Not Applicable

\section{Competing interests}

The authors declare that they have no competing interests.

\section{Author details}

${ }^{1}$ Department of Bioengineering, University of California-San Francisco Berkeley Joint Program, Room A-C106-B, 1 Irving St, San Francisco, CA 94143, USA. ${ }^{2}$ Department of Radiology and Biomedical Imaging, UC-San Francisco, San Francisco, CA 94143, USA. ${ }^{3}$ Kaiser Permanente Division of Research, Oakland, CA, USA. ${ }^{4}$ Accenture, San Francisco, CA 94143, USA. ${ }^{5}$ Applied Materials, Santa Clara, CA, USA. ${ }^{6}$ Research Advocate, UCSF Breast Science Advocacy Core, San Francisco, CA 94143, USA. 7 Departments of Medicine and Epidemiology and Biostatistics, UCSF, San Francisco, CA 94143, USA. ${ }^{8}$ Cancer Epidemiology, University of Hawaii Cancer Center, Honolulu, HI 96813, USA.

Received: 4 March 2019 Accepted: 13 June 2019

Published online: 22 June 2019

\section{References}

1. DeSantis C, Ma J, Bryan L, Jemal A. Breast cancer statistics, 2013: breast Cancer statistics, 2013. CA Cancer J Clin. 2014;64(1):52-62.

2. Narod SA. Tumour size predicts long-term survival among women with lymph node-positive breast cancer. Curr Oncol. 2012;19(5). [cited 2016 Sep 15]. Available from: http://www.current-oncology.com/index.php/ oncology/article/view/1043

3. Kerlikowske K. Comparative effectiveness of digital versus film-screen mammography in community practice in the United States: a cohort study. Ann Intern Med. 2011;155(8):493.

4. Kerlikowske K, Zhu W, Tosteson A, Sprague B, Tice J, Lehman C, et al. Identifying women with dense breasts at high risk for interval Cancer: a cohort study. Ann Intern Med. 2015;162(10):673-81.

5. Lehman CD, Arao RF, Sprague BL, Lee JM, Buist DSM, Kerlikowske K, et al. National Performance Benchmarks for modern screening digital mammography: update from the breast Cancer surveillance consortium. Radiology. 2017;283(1):49-58.

6. Kerlikowske K, Scott CG, Mahmoudzadeh AP, et al. Automated and clinical breast imaging reporting and data system density measures predict risk for screen-detected and interval cancers: a case-control study. Ann Intern Med. 2018;168(11):757-65.

7. Are You Dense. Available from: http://www.areyoudense.org/. [cited 25 Jun 2018].

8. Malkov S, Shepherd JA, Scott CG, Tamimi RM, Ma L, Bertrand KA, et al. Mammographic texture and risk of breast cancer by tumor type and estrogen receptor status. Breast Cancer Res [Internet]. 2016;18(1). [cited 2017 
Jul 13] Available from: http://breast-cancer-research.biomedcentral.com/ articles/10.1186/s13058-016-0778-1.

9. Strand F, Humphreys K, Cheddad A, Törnberg S, Azavedo E, Shepherd J, et al. Novel mammographic image features differentiate between interval and screen-detected breast cancer: a case-case study. Breast Cancer Res 2016; 18(1). [cited 2017 Sep 12] Available from: http://breast-cancer-research biomedcentral.com/articles/10.1186/s13058-016-0761-x.

10. Mainprize JG, Alonzo-Proulx O, Jong RA, Yaffe MJ. Quantifying masking in clinical mammograms via local detectability of simulated lesions. Med Phys. 2016;43(3):1249-58.

11. D'Orsi C, Sickles E, Mendelson E, Morris E, et al. ACR BI-RADS ${ }^{\oplus}$ atlas, breast imaging reporting and data system. Reston: American college of Radiology; 2013

12. Sechopoulos I. A review of breast tomosynthesis. Part I. the image acquisition process. Med Phys. 2013;40(1). Available from: https://www.ncbi. nlm.nih.gov/pmc/articles/PMC3548887/. [cited 19 Jul 2017].

13. Sechopoulos I. A review of breast tomosynthesis. Part II. Image reconstruction, processing and analysis, and advanced applications. Med Phys. 2013;40(1). Available from: https:/www.ncbi.nlm.nih.gov/pmc/articles/ PMC3548896/. [cited 2017 Jul 19].

14. O'Flynn EAM, Ledger AEW, deSouza NM. Alternative screening for dense breasts: MRI. Am J Roentgenol. 2015;204(2):W141-9.

15. Razek AAKA, Lattif MA, Denewer A, Farouk O, Nada N. Assessment of axillary lymph nodes in patients with breast cancer with diffusion-weighted MR imaging in combination with routine and dynamic contrast MR imaging. Breast Cancer. 2016;23(3):525-32.

16. Razek A, Zaki A, Bayoumi D, Taman S, AbdelWahab K, Algandour R. Diffusion tensor imaging parameters in differentiation recurrent breast cancer from post-operative changes in patients with breast-conserving surgery. Eur J Radiol. 2019;111:76-80.

17. Razek A, Gaballa G, Denewer A, Tawakol I. Diffusion weighted MR imaging of the breast. Acad Radiol. 2010;17:382-6.

18. Krizhevsky A, Sutskever I, Hinton GE. Imagenet classification with deep convolutional neural networks. In: Advances in neural information processing systems. 2012 [cited 2017 Oct 13]. p. 1097-1105. Available from: http://papers.nips.cc/paper/4824-imagenet-classification-with-deepconvolutional-neural-networks

19. He K, Zhang X, Ren S, Sun J. Deep residual learning for image recognition. CoRR. 2015

20. Long M, Wang J, Ding G, Pan SJ, Yu PS. Adaptation regularization: a general framework for transfer learning. IEEE Trans Knowl Data Eng. 2014;26(5):1076-89.

21. Greenspan H, van Ginneken B, Summers RM. Guest editorial deep learning in medical imaging: overview and future promise of an exciting new technique. IEEE Trans Med Imaging. 2016;35(5):1153-9.

22. Kourou K, Exarchos TP, Exarchos KP, Karamouzis MV, Fotiadis DI. Machine learning applications in cancer prognosis and prediction. Comput Struct Biotechnol J. 2015;13:8-17.

23. Achanta HK, Misganaw B, Vidyasagar M. Integrating biological data across multiple platforms using importance-weighted transfer learning and applications to breast cancer data sets. In: Control technology and applications (CCTA), 2017 IEEE conference on: IEEE; 2017. p. 955-60.

24. Ribli D, Horváth A, Unger Z, Pollner P, Csabai I. Detecting and classifying lesions in mammograms with deep learning. Sci Rep 2018;8(1). [cited 2018 Apr 2] Available from: http://www.nature.com/articles/s41598-018-22437-z.

25. Litjens $G$, Kooi T, Bejnordi BE, Setio AAA, Ciompi F, Ghafoorian M, et al. A survey on deep learning in medical image analysis. Med Image Anal. 2017;42:60-88.

26. Rajpurkar P, Irvin J, Zhu K, Yang B, Mehta H, Duan T, et al. CheXNet: radiologist-level pneumonia detection on chest $\mathrm{X}$-rays with deep. Learning. 2017;7.

27. Samala RK, Chan H-P, Hadjiiski LM, Helvie MA, Richter C, Cha KH. Crossdomain and multi-task transfer learning of deep convolutional neural network for breast cancer diagnosis in digital breast tomosynthesis. In: Mori K, Petrick N, editors. SPIE; 2018 [cited 2018 May 4]. p. 25. Available from: https://www.spiedigitallibrary.org/conference-proceedings-of-spie/10575/ 2293412/Cross-domain-and-multi-task-transfer-learning-of-deepconvolutional/10.1117/12.2293412.full

28. Simonyan K, Vedaldi A, Zisserman A. Deep Inside Convolutional Networks: Visualising Image Classification Models and Saliency Maps. ArXiv13126034 Cs. 2013[cited 2018 May 22]; Available from: http://arxiv.org/abs/1312.6034
29. Razzak MI, Naz S, Zaib A. Deep learning for medical image processing: overview, challenges and future. p. 30.

30. Abràmoff MD, Lou Y, Erginay A, Clarida W, Amelon R, Folk JC, et al. Improved automated detection of diabetic retinopathy on a publicly available dataset through integration of deep learning. Investig Opthalmology Vis Sci. 2016;57(13):5200.

31. Gulshan V, Peng L, Coram M, Stumpe MC, Wu D, Narayanaswamy A, et al. Development and validation of a deep learning algorithm for detection of diabetic retinopathy in retinal fundus photographs. JAMA. 2016;316(22):2402.

32. Burt JR, Torosdagli N, Khosravan N, RaviPrakash H, Mortazi A, Tissavirasingham F, et al. Deep learning beyond cats and dogs: recent advances in diagnosing breast cancer with deep neural networks. Br J Radiol. 2018;91:20170545.

33. de Moor T, Rodriguez-Ruiz A, Mérida AG, Mann R, Teuwen J. Automated soft tissue lesion detection and segmentation in digital mammography using a u-net deep learning network. ArXiv180206865 Cs. 2018 [cited 2018 Nov 25]; Available from: http://arxiv.org/abs/1802.06865

34. Teuwen J. Soft tissue lesion detection in mammography using deep neural networks for object detection; 2018. p. 9.

35. Aboutalib SS, Mohamed AA, Berg WA, Zuley ML, Sumkin JH, Wu S. Deep learning to distinguish recalled but benign mammography images in breast Cancer screening. Clin Cancer Res. 2018 [cited 2018 Nov 25]; Available from: http:// clincancerres.aacrjournals.org/lookup/doi/10.1158/1078-0432.CCR-18-1115

36. Lehman CD, Yala A, Schuster T, Dontchos B, Bahl M, Swanson K, et al. Mammographic breast density assessment using deep learning: clinical implementation. Radiology. 2018:180694.

37. Malkov S, Wang J, Shepherd J. Novel single x-ray absorptiometry method to solve for volumetric breast density in mammograms with paddle tilt. In: Hsieh J, Flynn MJ, editors. 2007 [cited 2016 Nov 7]. p. 651035. Available from: http:// proceedings.spiedigitallibrary.org/proceeding.aspx?doi=10.1117/12.710295

38. Ding Y, Sohn JH, Kawczynski MG, Trivedi H, Harnish R, Jenkins NW, et al. A deep learning model to predict a diagnosis of Alzheimer disease by using ${ }^{18}$ F-FDG PET of the brain. Radiology. 2018:180958.

39. Chollet F. Keras: deep learning library for theano and tensorflow; 2015.

40. Malkov S, Wang J, Kerlikowske K, Cummings SR, Shepherd JA. Single x-ray absorptiometry method for the quantitative mammographic measure of fibroglandular tissue volume. Med Phys. 2009:36(12):5525.

41. Holm J, Humphreys K, Li J, Ploner A, Cheddad A, Eriksson M, et al. Risk factors and tumor characteristics of interval cancers by mammographic density. J Clin Oncol. 2015;33(9):1030-7.

42. Strand F. Determinants of interval cancer and tumor size among breast cancer screening participants. 2018 [cited 2018 Jun 20]. Available from: http://hdl.handle.net/10616/46330

\section{Publisher's Note}

Springer Nature remains neutral with regard to jurisdictional claims in published maps and institutional affiliations.

Ready to submit your research? Choose BMC and benefit from:

- fast, convenient online submission

- thorough peer review by experienced researchers in your field

- rapid publication on acceptance

- support for research data, including large and complex data types

- gold Open Access which fosters wider collaboration and increased citations

- maximum visibility for your research: over $100 \mathrm{M}$ website views per year

At $\mathrm{BMC}$, research is always in progress.

Learn more biomedcentral.com/submissions 